\title{
Comparison Principle for Elliptic Equations with Mixed Singular Nonlinearities
}

\author{
Riccardo Durastanti ${ }^{1} \cdot$ Francescantonio Oliva $^{2}$
}

Received: 10 January 2020 / Accepted: 26 January 2021 / Published online: 20 February 2021

(C) The Author(s) 2021

\section{Abstract}

We deal with existence and uniqueness of positive solutions of an elliptic boundary value problem modeled by

$$
\begin{cases}-\Delta_{p} u=\frac{f}{u^{\gamma}}+g u^{q} & \text { in } \Omega, \\ u=0 & \text { on } \partial \Omega,\end{cases}
$$

where $\Omega$ is an open bounded subset of $\mathbb{R}^{N}, \Delta_{p} u:=\operatorname{div}\left(|\nabla u|^{p-2} \nabla u\right)$ is the usual $p$ Laplacian operator, $\gamma \geq 0$ and $0 \leq q \leq p-1 ; f$ and $g$ are nonnegative functions belonging to suitable Lebesgue spaces.

Keywords Nonlinear elliptic equations - Singular elliptic equations ·

Sublinear elliptic equations · Uniqueness

Mathematics Subject Classification (2010) 35J25 · 35J60 - 35J75 · 35A01 · 35A02

\section{Introduction}

In this paper we deal with an elliptic problem which simplest model is

$$
\begin{cases}-\Delta_{p} u=\frac{f}{u^{\gamma}}+g u^{q} & \text { in } \Omega, \\ u>0 & \text { in } \Omega, \\ u=0 & \text { on } \partial \Omega,\end{cases}
$$

Francescantonio Oliva

francescantonio.oliva@unina.it

Riccardo Durastanti

riccardo.durastanti@sbai.uniroma1.it

1 Dipartimento di Scienze di Base e Applicate per 1' Ingegneria, "Sapienza" Università di Roma, Via Scarpa 16, 00161, Roma, Italy

2 Dipartimento di Matematica e Applicazioni, Università di Napoli Federico II, Via Cintia, Monte S. Angelo, 80126, Napoli, Italy 
where $\Omega$ is an open bounded subset of $\mathbb{R}^{N}, \Delta_{p} u:=\operatorname{div}\left(|\nabla u|^{p-2} \nabla u\right)$ is the $p$-Laplacian operator $(1<p<N), \gamma, q \geq 0$ are such that $q<p-1$ or $q=p-1$, which correspond to the sublinear and to the linear behaviour in case $p=2$; here $f, g$ are nonnegative functions belonging to suitable Lebesgue spaces. Clearly the Dirichlet problem (1.1) is singular since the request that the solution is zero on the boundary of the set implies that the right hand side blows up. For (1.1) we are mainly interested to existence and uniqueness of possibly unbounded solutions with finite energy (i.e. $u \in W_{0}^{1, p}(\Omega)$ ).

Let us briefly recall the mathematical framework concerning problem (1.1); we start with the non-singular case, namely $f \equiv 0$.

The main idea of this paper comes from the seminal paper [9] where the authors show existence and uniqueness of a solution $u \in H_{0}^{1}(\Omega) \cap L^{\infty}(\Omega)$ to (1.1) in case $p=2, f \equiv 0$, $q<1$ and $g$ as a bounded nonnegative function. Let us also mention that classical arguments apply once that $u$ is bounded in order to get a $C^{1}$-solution, at least when the set $\Omega$ is smooth enough. Later, in [6], in presence of a possibly unbounded $g$ and if $q<p-1$, the existence of a solution is proven through an approximation process; here, even in the nonvariational case, it is proven existence of a solution with infinite energy (i.e. $u \notin W_{0}^{1, p}(\Omega)$ ) for rough data $g$.

Let us briefly underline that, when $p=2$, problem (1.1) with $f \equiv 0$ is strongly related to the porous media equation in the following way: if $u$ is a solution to (1.1) then for some positive constant $c, \tau>0$

$$
v(x, t)=c u(x)^{q}(t+\tau)^{\frac{-q}{1-q}},
$$

is a solution to

$$
g(x) u_{t}-\Delta v^{\frac{1}{q}}=0 .
$$

On the other side there is a huge literature concerning the purely singular equations, namely $g \equiv 0$. In presence of regular $f$ (say a positive $f \in C^{\eta}(\Omega)$ ), (1.1) was first treated in these pioneering works $[17,29,38]$; here the authors obtain existence and uniqueness of a classical solution (i.e. $u \in C^{2}(\Omega) \cap C(\bar{\Omega})$ ). Moreover, among other things, one has that: $u \in C^{2, \eta}(\Omega), u \notin C^{1}(\bar{\Omega})$ if $\gamma>1$ and $u \notin H_{0}^{1}(\Omega)$ if $\gamma \geq 3$. Furthermore we refer to [27] for more interesting results regarding the regularity of $u$.

For what concerns the weak theory of the purely singular case, existence of a distributional solution to (1.1) when the $f$ is only a nonnegative function in $L^{m}(\Omega)(m \geq 1)$ is established in [7]. This solution, if $\gamma \leq 1$ (i.e. themild singular case), attains the boundary datum in the classical sense of Sobolev traces; otherwise, when $\gamma>1$ (i.e. the strong singular case), only a power of the solution has zero Sobolev trace and the solution is shown to be locally in the same space. Later, in $[18,19,33]$, existence of solutions to (1.1) is given when the right hand side is of the general form $h(s) f$, with $h$ as a nonnegative and not necessarily monotone function such that $h(s) \leq s^{-\gamma}$ near zero and just bounded at infinity. For the nonhomogeneous case in which $q=0$ and $g \not \equiv 0$ we mention [32].

Dealing with uniqueness is more tricky; in [5] the authors show that the solution is unique in the class of $H_{0}^{1}(\Omega)$ and this kind of result has been extended to general nonincreasing nonlinearities and nonlinear operators in [31] for solutions in $W_{0}^{1, p}(\Omega)$. In [8], when $p=2$, the authors show that there is at most one solution to (1.1) belonging to $W_{0}^{1,1}(\Omega)$.

In [33], uniqueness of a distributional solution belonging to $W_{\text {loc }}^{1,1}(\Omega)$ (with suitable boundary conditions) is shown for a general measure datum and a nonincreasing nonlinearity. Finally in presence of a very general nonlinear operator and a nonincreasing $h$ it is shown in [19] the existence and uniqueness of a renormalized solution for a diffuse measure datum $f$. For further reading on singular problems we refer to [10-12, 21, 23, 24, 32] 
As one should expect the literature concerning (1.1) in presence of both $f$ and $g$ not identically zero is less investigated. Already in [38] the author proves existence of a classical solution to (1.1) when both $f$ and $g$ are regular enough, $p=2$ and $q<1$. In the same direction we refer to [15] where it is also investigated the superlinear case, which is a completely different framework. The uniqueness of classical solutions to (1.1) is shown in [36] in presence of the Laplacian operator and $q<p-1$; we also refer to [13] where, in case of regular $f$ and $g$ constant, it is proved existence and uniqueness of solutions to (1.1) if $q \leq 1$; here in the linear case it is proved existence under a smallness assumption on $g$ and nonnexistence otherwise. Then in [30], for $p>1$, through a sub and supersolution argument it is shown existence of solutions to (1.1) when the right hand side is of the form $h(u)+k(u)$ and no monotonicity is assumed on $h, k$. In [14] it is investigated the existence of a solution to (1.1) in case $p=2$ when $f$ and $g$ are functions in suitable Lebesgue spaces. Let us mention that in [35], for $p>1$, the authors show existence and uniqueness of finite energy solutions to (1.1) under suitable assumptions on $f, g$. We finally refer to $[22,26]$ for more interesting results.

The aim of this work is twofold. Firstly, we deal with uniqueness of finite energy solutions by employing the idea contained in [9]. More precisely we want to prove it for positive solutions to the Dirichlet problem associated to

$$
-\Delta_{p} u=F(x, u)
$$

where $p>1$ and $F$ is a Carathéodory function which is possibly unbounded both at the origin and at the infinity and such that

$$
F(x, s) s^{1-p} \text { decreases with respect to } s \text { for a.e. } x \in \Omega \text {. }
$$

Here the major difficult is dealing with a nonlinear operator when looking for comparison principles. Another issue which needs to be underlined is that the solutions are not required to be bounded; this implying the need of a suitable truncation arguments. It is also worth mentioning that (1.3) allows to deal with the case $q \leq p-1$, at least for positive $f$ if one considers the model case given by (1.1). This result is presented as the comparison principle given by Theorem 2.2 which, as a simple corollary, takes to uniqueness of finite energy solutions.

Other than uniqueness, we are interested to instances of finite energy solutions to (1.2); this is done both in the mild and in the strongly singular case by means of approximation arguments firstly if $q<p-1$; then we also give an existence result in case $q=p-1$. Summarizing, if $q<p-1$, we provide existence of finite energy solutions to equations as in $(1.1)$ if $g \in L^{\left(\frac{p^{*}}{1+q}\right)^{\prime}}(\Omega), \gamma \leq 1$ and $f \in L^{\left(\frac{p^{*}}{1-\gamma}\right)^{\prime}}(\Omega)$, where we mean $L^{1}(\Omega)$ once that $\gamma=1$.

Otherwise, we show that if $f \in L^{m}(\Omega)$ with $1<\gamma<2-\frac{1}{m}$ then the existence is guaranteed under the same assumptions on $g$. Let us also highlight that, as remarked in Section 3.2, there are instances in which one could expect finite energy solutions up to $\gamma<1+\frac{p(m-1)}{(p-1) m}$. Finally, once again if $f \in L^{\left(\frac{p^{*}}{1-\gamma}\right)^{\prime}}(\Omega)$, we also show the existence of a solution in case $q=p-1$ under a smallness assumption on $g$.

Let us mention that formally the change of variable $v=\frac{u^{\gamma+1}}{\gamma+1}$ for $p=2$ takes (1.1) to the following equation

$$
-\Delta v+\frac{\gamma}{\gamma+1} \frac{|\nabla v|^{2}}{v}=(\gamma+1)^{\frac{\gamma+\theta}{\gamma+1}} g v^{\frac{\gamma+\theta}{\gamma+1}}+f,
$$


which, for $g=0$, was extensively studied in the past, see for instance [1-3, 20, 25]. The previous discussion could be formalized and the existence and uniqueness results given in the current paper could provide information regarding problem (1.4).

The plan of the paper is the following: in Section 2 we state and prove the comparison principle and the associated uniqueness result for problems as in (1.1) (Theorem 2.2 and Corollary 2.3). In Section 3 we give some existence results; precisely we investigate both the mild and the strongly singular case when $q<p-1$ (Theorem 3.2 and Theorem 3.4); moreover we also treat a case in which $q=p-1$ (Theorem 3.5).

\subsection{Notation}

In the entire paper $\Omega$ is an open and bounded subset of $\mathbb{R}^{N}$, with $N \geq 1$. We denote by $\partial A$ the boundary and by $|A|$ the Lebesgue measure of a subset $A$ of $\mathbb{R}^{N}$. By $C_{c}^{k}(\Omega)$, with $k \geq 1$, we mean the space of $C^{k}$ functions with compact support in $\Omega$.

For any $q>1, q^{\prime}:=\frac{q}{q-1}$ is the Hölder conjugate exponent of $q$, while for any $1 \leq p<N$, $p^{*}=\frac{N p}{N-p}$ is the Sobolev conjugate exponent of $p$.

We denote by $\chi_{E}$ the characteristic function of $E \subset \Omega$, namely

$$
\chi_{E}(x)= \begin{cases}1 & x \in E, \\ 0 & x \in \Omega \backslash E,\end{cases}
$$

and by $f^{+}:=\max (f, 0), f^{-}:=-\min (f, 0)$ the positive and the negative part of a function $f$. We will widely use the following function defined for a fixed $k>0$ and $s \in \mathbb{R}$

$$
T_{k}(s)=\max (-k, \min (s, k)),
$$

and

$$
V_{\delta}(s)= \begin{cases}1 & s \leq \delta, \\ \frac{2 \delta-s}{\delta} & \delta<s<2 \delta, \\ 0 & s \geq 2 \delta .\end{cases}
$$

If no otherwise specified, we will denote by $C$ several constants whose value may change from line to line. These values will only depend on the data (for instance $C$ may depend on $\Omega, N$ and $p$ ) but they will never depend on the indexes of the sequences we will often introduce.

\section{Comparison Principle and Uniqueness}

Let $1<p<N$ and let us consider the following problem

$$
\begin{cases}-\Delta_{p} u=F(x, u) & \text { in } \Omega, \\ u>0 & \text { in } \Omega, \\ u=0 & \text { on } \partial \Omega,\end{cases}
$$

where the nonlinearity $F: \Omega \times(0, \infty) \rightarrow[0, \infty)$ is a general Carathéodory function. We start specifying the notion of weak solution to (2.1). 
Definition 2.1 A positive function $u \in W_{0}^{1, p}(\Omega)$ is a weak solution to $(2.1)$ if $F(x, u) \in$ $L_{\mathrm{loc}}^{1}(\Omega)$ and if

$$
\int_{\Omega}|\nabla u|^{p-2} \nabla u \cdot \nabla \varphi=\int_{\Omega} F(x, u) \varphi, \forall \varphi \in C_{c}^{1}(\Omega) .
$$

In order to deal with uniqueness of solutions, we present a comparison principle for solutions to (2.1) provided the right hand side enjoys some monotonicity condition. In particular let us consider $v_{1}, v_{2}$ solutions to

$$
\begin{cases}-\Delta_{p} v_{i}=G_{i}(x, v) & \text { in } \Omega, \\ v_{i}>0 & \text { in } \Omega, \\ v_{i}=0 & \text { on } \partial \Omega,\end{cases}
$$

where the nonlinearities $G_{1}, G_{2}: \Omega \times(0, \infty) \rightarrow[0, \infty)$ are Carathéodory functions. We state the main result of this section.

Theorem 2.2 (Comparison Principle) Let us assume $G_{1}, G_{2}$ are nonnegative functions such that either $G_{1}(x, s) s^{1-p}$ or $G_{2}(x, s) s^{1-p}$ is decreasing with respect to $s$ and for almost every $x \in \Omega$ and

$$
G_{1}(x, s) \leq G_{2}(x, s)
$$

for almost every $x \in \Omega$ and for all $s \in(0, \infty)$. Let $v_{1}$ and $v_{2}$ be weak solutions to problem (2.3) with data, respectively, $G_{1}, G_{2}$ then $v_{1} \leq v_{2}$ almost everywhere in $\Omega$.

As a simple corollary of the previous result, one has that uniqueness holds for weak solutions to (2.1).

Corollary 2.3 (Uniqueness) Let us assume that $F$ is a nonnegative function such that $F(x, s) s^{1-p}$ is decreasing with respect to $s$ and for almost every $x \in \Omega$. Then there exists at most one weak solution to problem (2.1).

Remark 2.4 Just to give an idea, Corollary 2.3 gives uniqueness of solutions to (2.1) when $F$ is modelled by

$$
F(x, s)=\frac{f(x)}{s^{\gamma}}+g(x) s^{q}, \text { with } f+g>0 \text { a.e. in } \Omega,
$$

or by

$$
F(x, s)=\frac{f(x)}{s^{\gamma}}+g(x) s^{p-1}, \text { with } f>0 \text { a.e. in } \Omega,
$$

where $f, g$ are nonnegative functions defined almost everywhere, $\gamma \geq 0$ and $0 \leq q<p-1$.

\subsection{Proof of the Comparison Principle}

In this section we prove the comparison principle for weak solutions to problem (2.1) and, as a consequence, we deduce the uniqueness result, namely Corollary 2.3. 
Proof of Theorem 2.2 First of all we need to show that for any weak solution $u$ to (2.1), the formulation (2.2) can be extended for $W^{1, p}$-test functions. We consider a nonnegative $\varphi \in W_{0}^{1, p}(\Omega)$ and a sequence of nonnegative functions $\varphi_{\eta, n} \in C_{c}^{1}(\Omega)$ such that

$$
\left\{\begin{array}{l}
\varphi_{\eta, n} \stackrel{\eta \rightarrow 0}{\rightarrow} \varphi_{n} \stackrel{n \rightarrow \infty}{\rightarrow} \varphi \text { in } W_{0}^{1, p}(\Omega) \\
\operatorname{supp} \varphi_{n} \subset \subset \Omega: 0 \leq \varphi_{n} \leq \varphi \text { for all } n \in \mathbb{N} .
\end{array}\right.
$$

An example of such $\varphi_{\eta, n}$ is $\rho_{\eta} *\left(\varphi \wedge \phi_{n}\right)\left(\varphi \wedge \phi_{n}:=\inf \left(\varphi, \phi_{n}\right)\right)$ where $\rho_{\eta}$ is a smooth mollifier and $\phi_{n}$ is a sequence of nonnegative functions in $C_{c}^{1}(\Omega)$ which converges to $\varphi$ in $W_{0}^{1, p}(\Omega)$.

Hence let us take $\varphi_{\eta, n}$ as a test function in (2.2), yielding to

$$
\int_{\Omega}|\nabla u|^{p-2} \nabla u \cdot \nabla \varphi_{\eta, n}=\int_{\Omega} F(x, u) \varphi_{\eta, n} .
$$

We want to pass first $\eta$ to zero and then $n$ to infinity in the previous.

Since $u \in W_{0}^{1, p}(\Omega)$ one can pass to the limit the first term recalling that $\varphi_{\eta, n}$ converges to $\varphi_{n}$ in $W_{0}^{1, p}(\Omega)$. For the right hand side one has that $F(x, u) \in L_{\text {loc }}^{1}(\Omega)$ that gives that we can pass $\eta \rightarrow 0$ since $\varphi_{\eta, n}$ converges $*$-weakly in $L^{\infty}(\Omega)$ to $\varphi_{n}$ which has compact support in $\Omega$. Hence we deduce

$$
\int_{\Omega}|\nabla u|^{p-2} \nabla u \cdot \nabla \varphi_{n}=\int_{\Omega} F(x, u) \varphi_{n} .
$$

Now let observe that by the Young inequality

$$
\int_{\Omega} F(x, u) \varphi_{n} \leq \int_{\Omega}|\nabla u|^{p}+\int_{\Omega}\left|\nabla \varphi_{n}\right|^{p},
$$

and by the Fatou Lemma with respect to $n$, one gets

$$
\int_{\Omega} F(x, u) \varphi \leq C .
$$

Now we take $n \rightarrow \infty$ in (2.5). For the term on the left hand side we can reason as already done when $\eta \rightarrow 0$. For the right hand side of (2.5) one can easily apply the Lebesgue Theorem since

$$
F(x, u) \varphi_{n} \leq F(x, u) \varphi \stackrel{(2.6)}{\in} L^{1}(\Omega),
$$

which gives

$$
\int_{\Omega}|\nabla u|^{p-2} \nabla u \cdot \nabla \varphi=\int_{\Omega} F(x, u) \varphi
$$

for every $\varphi \in W_{0}^{1, p}(\Omega)$.

Since $v_{1}$ and $v_{2}$ are weak solutions to problem (2.3) with data $G_{1}, G_{2}$ then, recalling (2.7), one can test both equations with $W_{0}^{1, p}$-functions. From here we suppose that $G_{1}(x, s) s^{1-p}$ is decreasing with respect to $s$ for almost every $x \in \Omega$; if one is in the other case, then slight modifications will be needed.

Let us fix $\varepsilon>0$ and $k \in \mathbb{N}$ and let us define

$$
A_{k, \varepsilon}:=\left\{x \in \Omega: 0 \leq\left(v_{1}(x)+\varepsilon\right)^{p}-\left(v_{2}(x)+\varepsilon\right)^{p} \leq k\right\}, \quad A_{k, \varepsilon}^{c}=\Omega \backslash A_{k, \varepsilon},
$$

and

$$
A_{k}=\left\{x \in \Omega: 0 \leq v_{1}(x)^{p}-v_{2}(x)^{p} \leq k\right\}, \quad A_{k}^{c}=\Omega \backslash A_{k} .
$$


We consider the following two functions:

$$
\psi_{1}=\frac{T_{k}\left(\left(\left(v_{1}+\varepsilon\right)^{p}-\left(v_{2}+\varepsilon\right)^{p}\right)^{+}\right)}{\left(v_{1}+\varepsilon\right)^{p-1}}, \quad \psi_{2}=\frac{T_{k}\left(\left(\left(v_{1}+\varepsilon\right)^{p}-\left(v_{2}+\varepsilon\right)^{p}\right)^{+}\right)}{\left(v_{2}+\varepsilon\right)^{p-1}}
$$

where $T_{k}$ is defined by (1.5). Let us also underline that $\psi_{1}, \psi_{2} \in W_{0}^{1, p}(\Omega)$ (see Remark 2.5 below). One has

$$
\begin{aligned}
\nabla \psi_{1}= & \left(\nabla v_{1}-p\left(\frac{v_{2}+\varepsilon}{v_{1}+\varepsilon}\right)^{p-1} \nabla v_{2}+(p-1)\left(\frac{v_{2}+\varepsilon}{v_{1}+\varepsilon}\right)^{p} \nabla v_{1}\right) \chi_{A_{k, \varepsilon}} \\
& -(p-1) \frac{T_{k}\left(\left(\left(v_{1}+\varepsilon\right)^{p}-\left(v_{2}+\varepsilon\right)^{p}\right)^{+}\right)}{\left(v_{1}+\varepsilon\right)^{p}} \nabla v_{1} \chi_{A_{k, \varepsilon}^{c}}
\end{aligned}
$$

and

$$
\begin{aligned}
\nabla \psi_{2}= & -\left(\nabla v_{2}-p\left(\frac{v_{1}+\varepsilon}{v_{2}+\varepsilon}\right)^{p-1} \nabla v_{1}+(p-1)\left(\frac{v_{1}+\varepsilon}{v_{2}+\varepsilon}\right)^{p} \nabla v_{2}\right) \chi_{A_{k, \varepsilon}} \\
& -(p-1) \frac{T_{k}\left(\left(\left(v_{1}+\varepsilon\right)^{p}-\left(v_{2}+\varepsilon\right)^{p}\right)^{+}\right)}{\left(v_{2}+\varepsilon\right)^{p}} \nabla v_{2} \chi_{A_{k, \varepsilon}^{c}} .
\end{aligned}
$$

We choose $\psi_{1}$ and $\psi_{2}$ as test functions in equations solved by, respectively, $v_{1}$ and $v_{2}$ and we subtract them yielding to

$$
\begin{aligned}
& \int_{A_{k, \varepsilon}}\left(\left|\nabla v_{1}\right|^{p}-\left(\frac{v_{1}+\varepsilon}{v_{2}+\varepsilon}\right)^{p}\left|\nabla v_{2}\right|^{p}-p\left(\frac{v_{1}+\varepsilon}{v_{2}+\varepsilon}\right)^{p-1}\left|\nabla v_{2}\right|^{p-2} \nabla v_{2} \cdot\left(\nabla v_{1}-\left(\frac{v_{1}+\varepsilon}{v_{2}+\varepsilon}\right) \nabla v_{2}\right)\right) \\
& +\int_{A_{k, \varepsilon}}\left(\left|\nabla v_{2}\right|^{p}-\left(\frac{v_{2}+\varepsilon}{v_{1}+\varepsilon}\right)^{p}\left|\nabla v_{1}\right|^{p}-p\left(\frac{v_{2}+\varepsilon}{v_{1}+\varepsilon}\right)^{p-1}\left|\nabla v_{1}\right|^{p-2} \nabla v_{1} \cdot\left(\nabla v_{2}-\left(\frac{v_{2}+\varepsilon}{v_{1}+\varepsilon}\right) \nabla v_{1}\right)\right) \\
& +(p-1) \int_{A_{k, \varepsilon}^{c}}\left(\frac{T_{k}\left(\left(\left(v_{1}+\varepsilon\right)^{p}-\left(v_{2}+\varepsilon\right)^{p}\right)^{+}\right)}{\left(v_{2}+\varepsilon\right)^{p}}\left|\nabla v_{2}\right|^{p}-\frac{T_{k}\left(\left(\left(v_{1}+\varepsilon\right)^{p}-\left(v_{2}+\varepsilon\right)^{p}\right)^{+}\right)}{\left(v_{1}+\varepsilon\right)^{p}}\left|\nabla v_{1}\right|^{p}\right) \\
& \leq \int_{\Omega}\left(\frac{G_{1}\left(x, v_{1}\right)}{\left(v_{1}+\varepsilon\right)^{p-1}}-\frac{G_{2}\left(x, v_{2}\right)}{\left(v_{2}+\varepsilon\right)^{p-1}}\right) T_{k}\left(\left(\left(v_{1}+\varepsilon\right)^{p}-\left(v_{2}+\varepsilon\right)^{p}\right)^{+}\right) .
\end{aligned}
$$

Now using the following classical estimate due to the convexity of the power function (recall that $p>1$ )

$$
|\xi|^{p}-|\eta|^{p}-p|\eta|^{p-2} \eta \cdot(\xi-\eta) \geq 0, \quad \forall \xi, \eta \in \mathbb{R}^{N}
$$

one has

$$
\begin{aligned}
& (p-1) \int_{A_{k, \varepsilon}^{c}} \frac{T_{k}\left(\left(\left(v_{1}+\varepsilon\right)^{p}-\left(v_{2}+\varepsilon\right)^{p}\right)^{+}\right)}{\left(v_{2}+\varepsilon\right)^{p}}\left|\nabla v_{2}\right|^{p} \\
& \leq(p-1) \int_{A_{k, \varepsilon}^{c}} \frac{T_{k}\left(\left(\left(v_{1}+\varepsilon\right)^{p}-\left(v_{2}+\varepsilon\right)^{p}\right)^{+}\right)}{\left(v_{1}+\varepsilon\right)^{p}}\left|\nabla v_{1}\right|^{p} \\
& +\int_{\Omega}\left(\frac{G_{1}\left(x, v_{1}\right)}{\left(v_{1}+\varepsilon\right)^{p-1}}-\frac{G_{2}\left(x, v_{2}\right)}{\left(v_{2}+\varepsilon\right)^{p-1}}\right) T_{k}\left(\left(\left(v_{1}+\varepsilon\right)^{p}-\left(v_{2}+\varepsilon\right)^{p}\right)^{+}\right)
\end{aligned}
$$


Noting that the first term of (2.9) is nonnegative, we have

$$
\begin{aligned}
0 & \leq(p-1) \int_{A_{k, \varepsilon}^{c}} \frac{T_{k}\left(\left(\left(v_{1}+\varepsilon\right)^{p}-\left(v_{2}+\varepsilon\right)^{p}\right)^{+}\right)}{\left(v_{1}+\varepsilon\right)^{p}}\left|\nabla v_{1}\right|^{p} \\
& +\int_{\Omega}\left(\frac{G_{1}\left(x, v_{1}\right)}{\left(v_{1}+\varepsilon\right)^{p-1}}-\frac{G_{2}\left(x, v_{2}\right)}{\left(v_{2}+\varepsilon\right)^{p-1}}\right) T_{k}\left(\left(\left(v_{1}+\varepsilon\right)^{p}-\left(v_{2}+\varepsilon\right)^{p}\right)^{+}\right) \\
& \quad \leq .4(p-1) \int_{A_{k, \varepsilon}^{c}} \frac{T_{k}\left(\left(\left(v_{1}+\varepsilon\right)^{p}-\left(v_{2}+\varepsilon\right)^{p}\right)^{+}\right)}{\left(v_{1}+\varepsilon\right)^{p}}\left|\nabla v_{1}\right|^{p} \\
& +\int_{\Omega}\left(\frac{G_{1}\left(x, v_{1}\right)}{\left(v_{1}+\varepsilon\right)^{p-1}}-\frac{G_{1}\left(x, v_{2}\right)}{\left(v_{2}+\varepsilon\right)^{p-1}}\right) T_{k}\left(\left(\left(v_{1}+\varepsilon\right)^{p}-\left(v_{2}+\varepsilon\right)^{p}\right)^{+}\right) .
\end{aligned}
$$

Denoting $r_{k, \varepsilon}, \tilde{r}_{k, \varepsilon}$ as follows

$$
\begin{aligned}
r_{k, \varepsilon}= & (p-1) \frac{T_{k}\left(\left(\left(v_{1}+\varepsilon\right)^{p}-\left(v_{2}+\varepsilon\right)^{p}\right)^{+}\right)}{\left(v_{1}+\varepsilon\right)^{p}}\left|\nabla v_{1}\right|^{p} \chi_{A_{k, \varepsilon}^{c}} \\
& +\left(\frac{G_{1}\left(x, v_{1}\right)}{\left(v_{1}+\varepsilon\right)^{p-1}}-\frac{G_{1}\left(x, v_{2}\right)}{\left(v_{2}+\varepsilon\right)^{p-1}}\right) T_{k}\left(\left(\left(v_{1}+\varepsilon\right)^{p}-\left(v_{2}+\varepsilon\right)^{p}\right)^{+}\right),
\end{aligned}
$$

and

$$
\begin{aligned}
\tilde{r}_{k, \varepsilon}= & (p-1) \frac{T_{k}\left(\left(\left(v_{1}+\varepsilon\right)^{p}-\left(v_{2}+\varepsilon\right)^{p}\right)^{+}\right)}{\left(v_{1}+\varepsilon\right)^{p}}\left|\nabla v_{1}\right|^{p} \chi_{A_{k, \varepsilon}^{c}} \\
& +\frac{G_{1}\left(x, v_{1}\right)}{\left(v_{1}+\varepsilon\right)^{p-1}} T_{k}\left(\left(\left(v_{1}+\varepsilon\right)^{p}-\left(v_{2}+\varepsilon\right)^{p}\right)^{+}\right),
\end{aligned}
$$

then one has

$$
0 \leq r_{k, \varepsilon}^{+} \leq \tilde{r}_{k, \varepsilon}
$$

Since $v_{1}, v_{2}$ are positive then one has that $r_{k, \varepsilon}^{+}\left(r_{k, \varepsilon}^{-}\right)$converges to $r_{k}^{+}\left(r_{k}^{-}\right.$resp.) and $\tilde{r}_{k, \varepsilon}$ converges to $\tilde{r}_{k}$ almost everywhere in $\Omega$, where

$$
r_{k}=(p-1) \frac{T_{k}\left(\left(v_{1}^{p}-v_{2}^{p}\right)^{+}\right)}{v_{1}^{p}}\left|\nabla v_{1}\right|^{p} \chi_{A_{k}^{c}}+\left(\frac{G_{1}\left(x, v_{1}\right)}{v_{1}^{p-1}}-\frac{G_{1}\left(x, v_{2}\right)}{v_{2}^{p-1}}\right) T_{k}\left(\left(v_{1}^{p}-v_{2}^{p}\right)^{+}\right),
$$

and

$$
\tilde{r}_{k}=(p-1) \frac{T_{k}\left(\left(v_{1}^{p}-v_{2}^{p}\right)^{+}\right)}{v_{1}^{p}}\left|\nabla v_{1}\right|^{p} \chi_{A_{k}^{c}}+\frac{G_{1}\left(x, v_{1}\right)}{v_{1}^{p-1}} T_{k}\left(\left(v_{1}^{p}-v_{2}^{p}\right)^{+}\right) .
$$

Moreover, using that $T_{k}(s) \leq s$ for $s \geq 0$, we deduce that

$$
\frac{T_{k}\left(\left(\left(v_{1}+\varepsilon\right)^{p}-\left(v_{2}+\varepsilon\right)^{p}\right)^{+}\right)}{\left(v_{1}+\varepsilon\right)^{p}} \chi_{A_{k, \varepsilon}^{c}} \leq 1,
$$

and

$$
\frac{T_{k}\left(\left(\left(v_{1}+\varepsilon\right)^{p}-\left(v_{2}+\varepsilon\right)^{p}\right)^{+}\right)}{\left(v_{1}+\varepsilon\right)^{p-1}} \leq \frac{\left(v_{1}+\varepsilon\right)^{p}-\varepsilon^{p}}{\left(v_{1}+\varepsilon\right)^{p-1}} \leq p v_{1},
$$

where the last inequality holds by means of the Langrange Theorem.

It follows that

$$
\tilde{r}_{k, \varepsilon} \stackrel{(2.12),(2.13)}{\leq}(p-1)\left|\nabla v_{1}\right|^{p}+p G_{1}\left(x, v_{1}\right) v_{1} .
$$


Since $v_{1} \in W_{0}^{1, p}(\Omega)$ and from (2.6) one has that the right hand side of (2.14) belongs to $L^{1}(\Omega)$. This implies, applying the Lebesgue Theorem, that $\tilde{r}_{k, \varepsilon}$ strongly converges to $\tilde{r}_{k}$ in $L^{1}(\Omega)$. Now starting from (2.11) and applying the Vitali Theorem, we obtain that

$$
r_{k, \varepsilon}^{+} \rightarrow r_{k}^{+} \text {strongly in } L^{1}(\Omega) \text {. }
$$

As regards $r_{k, \varepsilon}^{-}$, applying the Fatou Lemma, we have

$$
\limsup _{\varepsilon \rightarrow 0} \int_{\Omega}-r_{k, \varepsilon}^{-} \leq \int_{\Omega}-r_{k}^{-}
$$

Hence we deduce that

$$
0 \stackrel{(2.10)}{\leq} \limsup _{\varepsilon \rightarrow 0} \int_{\Omega} r_{k, \varepsilon}=\limsup _{\varepsilon \rightarrow 0} \int_{\Omega}\left(r_{k, \varepsilon}^{+}-r_{k, \varepsilon}^{-}\right) \stackrel{(2.15),(2.16)}{\leq} \int_{\Omega}\left(r_{k}^{+}-r_{k}^{-}\right)=\int_{\Omega} r_{k} .
$$

Thus, until now, we have shown that

$$
0 \leq \int_{\Omega}\left((p-1) \frac{T_{k}\left(\left(v_{1}^{p}-v_{2}^{p}\right)^{+}\right)}{v_{1}^{p}}\left|\nabla v_{1}\right|^{p} \chi_{A_{k}^{c}}+\left(\frac{G_{1}\left(x, v_{1}\right)}{v_{1}^{p-1}}-\frac{G_{1}\left(x, v_{2}\right)}{v_{2}^{p-1}}\right) T_{k}\left(\left(v_{1}^{p}-v_{2}^{p}\right)^{+}\right)\right) .
$$

Now we pass to the limit in (2.17) as $k$ tends to infinity. We note that $\chi_{A_{k}^{c}}$ tends to 0 as $k$ tends to infinity. Moreover, using (2.12) with $\varepsilon=0$, we have

$$
\frac{T_{k}\left(\left(v_{1}^{p}-v_{2}^{p}\right)^{+}\right)}{v_{1}^{p}}\left|\nabla v_{1}\right|^{p} \chi_{A_{k}^{c}} \leq\left|\nabla v_{1}\right|^{p} \in L^{1}(\Omega),
$$

since $v_{1} \in W_{0}^{1, p}(\Omega)$. This implies, applying the Lebesgue Theorem, that

$$
\frac{T_{k}\left(\left(v_{1}^{p}-v_{2}^{p}\right)^{+}\right)}{v_{1}^{p}}\left|\nabla v_{1}\right|^{p} \chi_{A_{k}^{c}} \rightarrow 0 \text { strongly in } L^{1}(\Omega) .
$$

As regards the second term in the right hand side of (2.17), from $G_{1}(x, s) s^{1-p}$ decreasing with respect to $s$, one has that

$$
0 \leq-\left(\frac{G_{1}\left(x, v_{1}\right)}{v_{1}^{p-1}}-\frac{G_{1}\left(x, v_{2}\right)}{v_{2}^{p-1}}\right) T_{k}\left(\left(v_{1}^{p}-v_{2}^{p}\right)^{+}\right),
$$

where the right hand side of (2.19) is increasing in $k$. Applying Beppo Levi's Theorem, we obtain that

$$
\lim _{k \rightarrow \infty} \int_{\Omega}\left(\frac{G_{1}\left(x, v_{1}\right)}{v_{1}^{p-1}}-\frac{G_{1}\left(x, v_{2}\right)}{v_{2}^{p-1}}\right) T_{k}\left(\left(v_{1}^{p}-v_{2}^{p}\right)^{+}\right)=\int_{\Omega}\left(\frac{G_{1}\left(x, v_{1}\right)}{v_{1}^{p-1}}-\frac{G_{1}\left(x, v_{2}\right)}{v_{2}^{p-1}}\right)\left(v_{1}^{p}-v_{2}^{p}\right)^{+} .
$$

By passing to the limit as $k$ tends to infinity in (2.17), using (2.18) and (2.20), we have

$$
0 \leq \int_{\Omega}\left(\frac{G_{1}\left(x, v_{1}\right)}{v_{1}^{p-1}}-\frac{G_{1}\left(x, v_{2}\right)}{v_{2}^{p-1}}\right)\left(v_{1}^{p}-v_{2}^{p}\right)^{+} .
$$

Furthermore from the fact that $G_{1}(x, s) s^{1-p}$ is decreasing with respect to $s$, one yields to

$$
\left(\frac{G_{1}\left(x, v_{1}\right)}{v_{1}^{p-1}}-\frac{G_{1}\left(x, v_{2}\right)}{v_{2}^{p-1}}\right)\left(v_{1}^{p}-v_{2}^{p}\right)^{+} \leq 0 \quad \text { a.e. in } \Omega,
$$

which, gathered with (2.21), gives that $\left(v_{1}^{p}-v_{2}^{p}\right)^{+} \equiv 0$, that is $v_{1} \leq v_{2}$ almost everywhere in $\Omega$. 
Remark 2.5 Here we show that $\psi_{1}, \psi_{2}$ defined by (2.8) belong to $W_{0}^{1, p}(\Omega)$. We focus on $\psi_{2}$. As a consequence of Lemma 1.1 contained in [37] and the fact the $v_{1}, v_{2}$ have finite energy, we have that the function $\psi_{h}$ defined as

$$
\psi_{h}=\frac{T_{k}\left(\left(\left(v_{1}+\varepsilon\right)^{p}-\left(T_{h}\left(v_{2}+\varepsilon\right)\right)^{p}\right)^{+}\right)}{\left(v_{2}+\varepsilon\right)^{p-1}}
$$

belongs to $W_{0}^{1, p}(\Omega)$ for every $h \geq 0$. Moreover, by computing its gradient, we get

$$
\begin{aligned}
\nabla \psi_{h}= & -p \nabla v_{2} \chi_{\left\{v_{2}+\varepsilon \leq h\right\} \cap A_{k, \varepsilon, h} \cap B_{h}}+p\left(\frac{v_{1}+\varepsilon}{v_{2}+\varepsilon}\right)^{p-1} \nabla v_{1} \chi_{A_{k, \varepsilon, h} \cap B_{h}} \\
& -(p-1) \frac{T_{k}\left(\left(\left(v_{1}+\varepsilon\right)^{p}-\left(T_{h}\left(v_{2}+\varepsilon\right)\right)^{p}\right)^{+}\right)}{\left(v_{2}+\varepsilon\right)^{p}} \nabla v_{2},
\end{aligned}
$$

where

$$
A_{k, \varepsilon, h}=\left\{x \in \Omega: 0 \leq\left(v_{1}(x)+\varepsilon\right)^{p}-\left(T_{h}\left(v_{2}(x)+\varepsilon\right)\right)^{p} \leq k\right\}
$$

and

$$
B_{h}=\left\{x \in \Omega: v_{1}(x)+\varepsilon \geq T_{h}\left(v_{2}(x)+\varepsilon\right)\right\} .
$$

It follows from the definition of $A_{k, \varepsilon, h}$ that

$$
\left(\frac{v_{1}+\varepsilon}{v_{2}+\varepsilon}\right)^{p-1} \leq\left(\frac{k}{\varepsilon^{p}}+1\right)^{\frac{p-1}{p}}
$$

This implies that

$$
\left|\nabla \psi_{h}\right|^{p} \leq C(p, k, \varepsilon)\left(\left|\nabla v_{2}\right|^{p}+\left|\nabla v_{1}\right|^{p}\right)
$$

with $C(p, k, \varepsilon)$ a positive constant dependent only on $p, k, \varepsilon$. Hence, using $v_{1}, v_{2} \in$ $W_{0}^{1, p}(\Omega)$, we deduce that $\left\{\psi_{h}\right\}$ is bounded in $W_{0}^{1, p}(\Omega)$ uniformly in $h$. Moreover $\psi_{h}$ converges to $\psi_{2}$ almost everywhere in $\Omega$. So that $\psi_{h}$ converges to $\psi_{2}$ weakly in $W_{0}^{1, p}(\Omega)$ and $\psi_{2}$ belongs to $W_{0}^{1, p}(\Omega)$. As regards $\varphi$, in a similar way it is possible to prove that $\psi_{1}$ belongs to $W_{0}^{1, p}(\Omega)$.

\section{Existence Results in Some Model Equations}

In this section we give existence results to (2.1) for some explicit nonlinearities $F$ of the following form

$$
F(x, s)=f(x) h(s)+g(x) k(s),
$$

where $f, g$ are nonnegative functions belonging to suitable Lebesgue space, with $f \not \equiv 0$, and $h, k:(0, \infty) \rightarrow[0, \infty)$ are continuous nonnegative functions such that

$$
\exists \gamma \geq 0, \underline{C}>0: h(s) \leq \frac{\underline{C}}{s^{\gamma}} \quad \forall s \in(0, \infty),
$$

and

$$
\exists q \geq 0, \bar{C}>0: k(s) \leq \bar{C} s^{q} \quad \forall s \in(0, \infty)
$$

Remark 3.1 Let us observe that (3.3) implies that $k$ can be extended by continuity at 0 defining $k(0)=0$.

We underline that we are not assuming any kind of monotonicity on the functions $h, k$ but just some control from the above. Moreover, the case of continuous and bounded $h, k$ are well contained in our existence result. 
For the sake of clarity we reformulate the problem under the assumption (3.1):

$$
\begin{cases}-\Delta_{p} u=f(x) h(u)+g(x) k(u) & \text { in } \Omega, \\ u>0 & \text { in } \Omega, \\ u=0 & \text { on } \partial \Omega .\end{cases}
$$

At first we state an existence result in case $\gamma \leq 1$ and $q<p-1$, which we recall that corresponds to the sublinear case when $p=2$; let us explicitly note that in the sequel we define $\left(\frac{p^{*}}{1-\gamma}\right)^{\prime}:=1$ if $\gamma=1$.

In particular one has the following result.

Theorem 3.2 Let $f \in L^{\left(\frac{p^{*}}{1-\gamma}\right)^{\prime}}(\Omega)$ be a nonnegative function not identically zero and let $g \in L^{\left(\frac{p^{*}}{1+q}\right)^{\prime}}(\Omega)$ be a nonnegative function. Let $h$ and $k$ be nonnegative continuous functions satisfying (3.2) with $\gamma \leq 1$ and (3.3) with $q<p-1$ respectively. Then there exists at least one weak solution to problem (3.4).

Remark 3.3 In the case $f \equiv 0$, if $k$ is an increasing function satisfying (3.3), the existence of a weak solution to (3.4) is contained in [6].

Next we deal with the more difficult case of a strong singularity; here, in order to deduce an existence result, we need some regularity on the $\Omega$.

Theorem 3.4 Let $\Omega$ satisfy the interior ball condition and let $f \in L^{m}(\Omega)$ with $m>1$ be a nonnegative function and let $g \in L^{\left(\frac{p^{*}}{1+q}\right)^{\prime}}(\Omega)$ be a nonnegative function. Let $h$ and $k$ be nonnegative continuous functions satisfying (3.2) with $1<\gamma<2-\frac{1}{m}$ and (3.3) with $q<p-1$ respectively. Then there exists at least one weak solution to problem (3.4).

Finally we also dealt with $q=p-1$. In the next result we denote by $C_{p}$ the best constant for the Poincaré inequality in $\Omega$; we also recall that $\bar{C}$ is the one defined by (3.3).

Theorem 3.5 Let $f \in L^{\left(\frac{p^{*}}{1-\gamma}\right)^{\prime}}(\Omega)$ be a nonnegative function not identically zero and let $g$ such that $\|g\|_{L^{\infty}(\Omega)}<\left(\bar{C} C_{p}^{p}\right)^{-1}$. Let $h$ and $k$ be nonnegative continuous functions satisfying (3.2) with $\gamma \leq 1$ and (3.3) with $q=p-1$ respectively. Then there exists at least one weak solution to problem (3.4).

Remark 3.6 Collecting the existence results contained in Theorems 3.2, 3.4 and 3.5 with the uniqueness result contained in Corollary 2.3 we obtain that there exists a unique solution $u \in W_{0}^{1, p}(\Omega)$ to

$$
-\Delta_{p} u=F(x, u)
$$

under the assumptions of Theorems 3.2 and 3.4 in case $(h(s)+k(s)) s^{1-p}$ is decreasing with respect to $s$ and requiring that $f+g$ is almost everywhere positive in $\Omega$.

Moreover under the assumptions of Theorem 3.5 one has a unique solution if $h(s) s^{1-p}$ is decreasing with respect to $s$ and $f$ is almost everywhere positive in $\Omega$. 


\subsection{Proof of the Existence Results}

Let us introduce the following scheme of approximation

$$
\begin{cases}-\Delta_{p} u_{n}=f_{n} h_{n}\left(u_{n}\right)+g_{n} k_{n}\left(u_{n}\right) & \text { in } \Omega, \\ u_{n}=0 & \text { on } \partial \Omega,\end{cases}
$$

where $f_{n}=T_{n}(f)$ and $g_{n}=T_{n}(g)$. Moreover, defining $h(0):=\lim _{s \rightarrow 0} h(s)$, we set

$$
h_{n}(s)=\left\{\begin{array}{ll}
T_{n}(h(s)) & \text { for } s>0, \\
\min (n, h(0)) & \text { otherwise, }
\end{array} \quad \text { and } \quad k_{n}(s)= \begin{cases}T_{n}(k(s)) & \text { for } s>0, \\
0 & \text { otherwise }\end{cases}\right.
$$

The existence of a weak solution $u_{n} \in W_{0}^{1, p}(\Omega)$ is guaranteed by [28]. Moreover, by Theorem 4.2 of [37], we get that $u_{n}$ is bounded and, since the right hand side of (3.5) is nonnegative, that $u_{n}$ is nonnegative.

Remark 3.7 Under the assumptions of Remark 3.6 one has that the approximating sequence $\left\{u_{n}\right\}$ is increasing w.r.t. $n$. Indeed defining $F_{n}(x, s)=f_{n}(x) h_{n}(s)+g_{n}(x) k_{n}(s)$ one deduces that for every $n$ in $\mathbb{N}$

$$
F_{n}(x, s) \leq F_{n+1}(x, s) \quad \forall s \in(0, \infty) \text { and for a.e. } x \in \Omega .
$$

This allows to apply Theorem 2.2 , yielding to

$$
u_{n} \leq u_{n+1} \quad \forall n \in \mathbb{N} .
$$

Proof of Theorem 3.2 We divide the proof in two steps. In the first one, we show a priori estimates on $u_{n}$, solutions to (3.5). In the second one we pass to the limit our approximation in order to deduce the existence of a weak solution to (3.4).

Step 1. Let us choose $u_{n}$ as a test function in the weak formulation of (3.5) and from the Hölder inequality and from EqS. 3.2, 3.3, one gets

$$
\begin{aligned}
\int_{\Omega}\left|\nabla u_{n}\right|^{p} & =\int_{\Omega}\left(f_{n} h_{n}\left(u_{n}\right) u_{n}+g_{n} k_{n}\left(u_{n}\right) u_{n}\right) \\
& \leq \underline{C} \int_{\Omega} f_{n} u_{n}^{1-\gamma}+\bar{C} \int_{\Omega} g_{n} u_{n}^{1+q} \\
& \leq \underset{L}{ }|| f\left\|_{L}\left(\frac{p^{*}}{1-\gamma}\right)_{(\Omega)}^{\prime}\right\| u_{n}\left\|_{L^{p^{*}}(\Omega)}^{1-\gamma}+\bar{C}\right\| g\left\|_{L}^{\left(\frac{p^{*}}{q+1}\right)^{\prime}}\right\| u_{(\Omega)} \|_{L^{p^{*}}(\Omega)}^{q+1} .
\end{aligned}
$$

If $\left\|u_{n}\right\|_{L^{p^{*}}(\Omega)} \leq 1$, we deduce that $\left\{u_{n}\right\}$ is bounded in $W_{0}^{1, p}(\Omega)$ uniformly in $n$. Otherwise, recalling that $0 \leq 1-\gamma<q+1<p$, we obtain, applying the Sobolev embedding Theorem on the left-hand side of (3.6), that

$$
\left\|u_{n}\right\|_{L^{p^{*}(\Omega)}}^{p} \leq C\left(\|f\|_{L}\left(\frac{p^{*}}{1-\gamma}\right)_{(\Omega)}^{\prime}+\|g\|_{L}\left(\frac{p^{*}}{q+1}\right)_{(\Omega)}^{\prime}\right)\left\|u_{n}\right\|_{L^{p^{*}(\Omega)}}^{q+1} .
$$

This implies, dividing by $\left\|u_{n}\right\|_{L^{p^{*}(\Omega)}}^{q+1}$ both members of (3.7), that $\left\{u_{n}\right\}$ is bounded in $L^{p^{*}}(\Omega)$ uniformly in $n$. It follows from (3.6) that $\left\{u_{n}\right\}$ is bounded in $W_{0}^{1, p}(\Omega)$ with respect to $n$. This implies that there exists a nonnegative function $u$ in $W_{0}^{1, p}(\Omega)$ such that $u_{n} \rightarrow u$ weakly in $W_{0}^{1, p}(\Omega)$ and almost everywhere in $\Omega$. Let 
us take $0 \leq \varphi \in W_{0}^{1, p}(\Omega)$ as test function in the weak formulation of (3.5); one obtains, using the Young inequality, that

$$
\int_{\Omega}\left(f_{n} h_{n}\left(u_{n}\right)+g_{n} k_{n}\left(u_{n}\right)\right) \varphi=\int_{\Omega}\left|\nabla u_{n}\right|^{p-2} \nabla u_{n} \cdot \nabla \varphi \leq \frac{1}{p^{\prime}} \int_{\Omega}\left|\nabla u_{n}\right|^{p}+\frac{1}{p} \int_{\Omega}|\nabla \varphi|^{p} \leq C .
$$

Hence $\left\{f_{n} h_{n}\left(u_{n}\right)+g_{n} k_{n}\left(u_{n}\right)\right\}$ is bounded in $L_{\text {loc }}^{1}(\Omega)$ and, applying Theorem 2.1 of [4], that $\nabla u_{n}$ converges almost everywhere in $\Omega$ to $\nabla u$.

Step 2. In this second step we prove that $u$ obtained in the first step is a weak solution to (3.4).

First of all we apply the Fatou Lemma in (3.8) in order to deduce that

$$
\int_{\Omega}(f h(u)+g k(u)) \varphi \leq \liminf _{n \rightarrow \infty} \int_{\Omega}\left(f_{n} h_{n}\left(u_{n}\right)+g_{n} k_{n}\left(u_{n}\right)\right) \varphi \leq C,
$$

hence $(f h(u)+g k(u)) \varphi \in L^{1}(\Omega)$ for any nonnegative $\varphi \in W_{0}^{1, p}(\Omega)$. As a consequence, if $h(s)$ is unbounded as $s$ tends to 0 , we deduce that

$$
\{u=0\} \subset\{f=0\},
$$

up to a set of zero Lebesgue measure.

From now on, we assume that $h(s)$ is unbounded as $s$ tends to 0 . Let $\varphi$ be a nonnegative function in $W_{0}^{1, p}(\Omega) \cap L^{\infty}(\Omega)$. Choosing it as test function in the weak formulation of (3.5) we have

$$
\int_{\Omega}\left|\nabla u_{n}\right|^{p-2} \nabla u_{n} \cdot \nabla \varphi=\int_{\Omega}\left(f_{n} h_{n}\left(u_{n}\right)+g_{n} k_{n}\left(u_{n}\right)\right) \varphi .
$$

We want to pass to the limit in (3.10) as $n$ tends to infinity. We fix $\delta>0$ and we decompose the right hand side in the following way:

$$
\begin{aligned}
\int_{\Omega}\left(f_{n} h_{n}\left(u_{n}\right)+g_{n} k_{n}\left(u_{n}\right)\right) \varphi= & \int_{\left\{u_{n} \leq \delta\right\}}\left(f_{n} h_{n}\left(u_{n}\right)+g_{n} k_{n}\left(u_{n}\right)\right) \varphi \\
& +\int_{\left\{u_{n}>\delta\right\}}\left(f_{n} h_{n}\left(u_{n}\right)+g_{n} k_{n}\left(u_{n}\right)\right) \varphi .
\end{aligned}
$$

Therefore we have, thanks to Lemma 1.1 contained in [37], that $V_{\delta}\left(u_{n}\right) \varphi$ belongs to $W_{0}^{1, p}(\Omega)$, where $V_{\delta}(s)$ is defined by (1.6). So we take it as test function in the weak formulation of (3.5) and we obtain

$$
\begin{aligned}
\int_{\left\{u_{n} \leq \delta\right\}}\left(f_{n} h_{n}\left(u_{n}\right)+g_{n} k_{n}\left(u_{n}\right)\right) \varphi & \stackrel{(1.6)}{\leq} \int_{\Omega}\left(f_{n} h_{n}\left(u_{n}\right)+g_{n} k_{n}\left(u_{n}\right)\right) V_{\delta}\left(u_{n}\right) \varphi \\
& =\int_{\Omega}\left|\nabla u_{n}\right|^{p-2} \nabla u_{n} \cdot \nabla \varphi V_{\delta}\left(u_{n}\right)-\frac{1}{\delta} \int_{\left\{\delta<u_{n}<2 \delta\right\}}\left|\nabla u_{n}\right|^{p} \varphi \\
& \leq \int_{\Omega}\left|\nabla u_{n}\right|^{p-2} \nabla u_{n} \cdot \nabla \varphi V_{\delta}\left(u_{n}\right)
\end{aligned}
$$

Using that $V_{\delta}$ is bounded we deduce that $\left|\nabla u_{n}\right|^{p-2} \nabla u_{n} V_{\delta}\left(u_{n}\right)$ converges to $|\nabla u|^{p-2} \nabla u V_{\delta}(u)$ weakly in $L^{p^{\prime}}(\Omega)^{N}$ as $n$ tends to infinity. This implies that

$$
\lim _{n \rightarrow \infty} \int_{\left\{u_{n} \leq \delta\right\}}\left(f_{n} h_{n}\left(u_{n}\right)+g_{n} k_{n}\left(u_{n}\right)\right) \varphi \leq \int_{\Omega}|\nabla u|^{p-2} \nabla u \cdot \nabla \varphi V_{\delta}(u) .
$$


Since $V_{\delta}(u)$ converges to $\chi_{\{u=0\}}$ a.e. in $\Omega$ as $\delta$ tends to 0 and since $u \in W_{0}^{1, p}(\Omega)$, then $|\nabla u|^{p-2} \nabla u \cdot \nabla \varphi V_{\delta}(u)$ converges to 0 a.e. in $\Omega$ as $\delta$ tends to 0 . Applying the Lebesgue Theorem on the right hand side of (3.12) we obtain that

$$
\lim _{\delta \rightarrow 0^{+}} \lim _{n \rightarrow \infty} \int_{\left\{u_{n} \leq \delta\right\}}\left(f_{n} h_{n}\left(u_{n}\right)+g_{n} k_{n}\left(u_{n}\right)\right) \varphi=0 .
$$

As regards the second term in the right hand side of (3.11) we have

$$
0 \leq\left(f_{n} h_{n}\left(u_{n}\right)+g_{n} k_{n}\left(u_{n}\right)\right) \chi_{\left\{u_{n}>\delta\right\}} \stackrel{(32),(3.3)}{\leq}\left(f \sup _{\{s>\delta\}} h(s)+\bar{C} g u_{n}^{q}\right) \varphi .
$$

Thanks to the a priori estimates on $u_{n}$ and using the Rellich-Kondrakov Theorem, we deduce, up to subsequence, that $u_{n}^{q}$ converges to $u^{q}$ strongly in $L^{\left(\frac{p^{*}}{1+q}\right)}(\Omega)$. Since $g$ belongs to $L^{\left(\frac{p^{*}}{1+q}\right)^{\prime}}(\Omega)$ this implies that the right hand side of (3.14) converges strongly in $L^{1}(\Omega)$. Moreover we can always assume that $\delta \notin\{\alpha:|\{u=\alpha\}|>0\}$ which is at most a countable set. As a consequence $\chi_{\left\{u_{n}>\delta\right\}}$ converges to $\chi_{\{u>\delta\}}$ a.e. in $\Omega$. Hence, using once again the Lebesgue Theorem in (3.14), we deduce first that $\left(f_{n} h_{n}\left(u_{n}\right)+g_{n} k_{n}\left(u_{n}\right)\right) \chi_{\left\{u_{n}>\delta\right\}} \varphi$ converges to $(f h(u)+g k(u)) \chi_{\{u>\delta\}} \varphi$ strongly in $L^{1}(\Omega)$ as $n$ tends to infinity, then, since $(f h(u)+g k(u)) \varphi$ belongs to $L^{1}(\Omega)$, that $(f h(u)+g k(u)) \chi_{\{u>\delta\}} \varphi$ converges to $(f h(u)+g k(u)) \chi_{\{u>0\}} \varphi$ strongly in $L^{1}(\Omega)$ as $\delta$ tends to 0. Recalling (3.9) and Remark 3.1, we conclude that

$$
\begin{aligned}
\lim _{\delta \rightarrow 0^{+}} \lim _{n \rightarrow \infty} \int_{\left\{u_{n}>\delta\right\}}\left(f_{n} h_{n}\left(u_{n}\right)+g_{n} k_{n}\left(u_{n}\right)\right) \varphi & =\int_{\{u>0\}}(f h(u)+g k(u)) \varphi \\
& \stackrel{(39))}{=} \int_{\Omega}(f h(u)+g k(u)) \varphi .
\end{aligned}
$$

Finally, using the weak convergence of $u_{n}$ in $W_{0}^{1, p}(\Omega)$ and the almost everywhere convergence of the gradients one can pass to the limit as $n \rightarrow \infty$ in the left hand side of (3.10). Moreover, by (3.13) and by (3.15), we can also take to the limit the right hand side of (3.10) in order to deduce that

$$
\int_{\Omega}|\nabla u|^{p-2} \nabla u \cdot \nabla \varphi=\int_{\Omega}(f h(u)+g k(u)) \varphi \quad \forall 0 \leq \varphi \in W_{0}^{1, p}(\Omega) \cap L^{\infty}(\Omega) .
$$

Moreover, decomposing any $\varphi=\varphi^{+}-\varphi^{-}$, and using that (3.16) is linear in $\varphi$, we deduce that (3.16) holds for every $\varphi \in W_{0}^{1, p}(\Omega) \cap L^{\infty}(\Omega)$.

We treated $h(s)$ unbounded as $s$ tends to 0 , as regards bounded function $h$ the proof is easier and the only difference deals with the passage to the limit in the right hand side of (3.10). We can avoid introducing $\delta$ and we can substitute (3.14) with

$$
0 \leq\left(f_{n} h_{n}\left(u_{n}\right)+g_{n} k_{n}\left(u_{n}\right)\right) \varphi \leq\left(f\|h\|_{L^{\infty}(\Omega)}+\bar{C} g u_{n}^{q}\right) \varphi .
$$

Using the same argument above we have that $\left(f_{n} h_{n}\left(u_{n}\right)+g_{n} k_{n}\left(u_{n}\right)\right) \varphi$ converges to $(f h(u)+g k(u)) \varphi$ strongly in $L^{1}(\Omega)$ as $n$ tends to infinity. Then we can conclude as in case of an unbounded $h$.

Finally, it follows from (3.16) and using the strong maximum principle that $u>0$ almost everywhere in $\Omega$. This implies that $u$ is a weak solution to (3.5).

Now we prove Theorem 3.4, namely the case where $\gamma>1$; here we need a more refined argument in order to control the possibly singular term. 
Proof of Theorem 3.4 We take $u_{n}$ as a test function in (3.5) yielding to

$$
\begin{aligned}
\int_{\Omega}\left|\nabla u_{n}\right|^{p} & \leq \int_{\Omega}\left(f_{n} h_{n}\left(u_{n}\right) u_{n}+g_{n} k_{n}\left(u_{n}\right) u_{n}\right) \leq \underline{C} \int_{\Omega} f_{n} u_{n}^{1-\gamma}+\bar{C} \int_{\Omega} g_{n} u_{n}^{1+q} \\
& \leq \underline{C} \int_{\Omega} f_{n} u_{n}^{1-\gamma}+\bar{C}\|g\|_{L}\left(\frac{p^{*}}{q+1}\right)^{\prime}\left\|u_{n}\right\|_{L^{p^{*}}(\Omega)}^{q+1} .
\end{aligned}
$$

Hence, we just need an estimate on the first term of the right hand side of (3.17). First of all let us observe that there exists a nonincreasing and continuous function $\underline{h}:[0, \infty) \rightarrow$ $[0, \infty)$ such that

$$
\underline{h}(s) \leq h_{n}(s), \quad \forall s>0, n \in \mathbb{N} .
$$

For the construction of such $\underline{h}$ we refer to [18]. Hence let us consider $v_{n} \in W_{0}^{1, p}(\Omega) \cap$ $L^{\infty}(\Omega)$ solution to

$$
\begin{cases}-\Delta_{p} v_{n}=\underline{h}\left(v_{n}\right) f_{n} & \text { in } \Omega, \\ v_{n}=0 & \text { on } \partial \Omega .\end{cases}
$$

Once again, reasoning as in $[18,19]$, one has that $v_{n}$ is nondecreasing with respect to $n$ and also that $u_{n} \geq v_{n} \geq v_{1}$. Moreover, it follows from the Hopf Lemma (see Lemma A.3 of [34]) that

$$
v_{1}(x) \geq C \delta(x), \text { for } x \in \Omega,
$$

where $\delta(x)$ is the distance function from the boundary $\partial \Omega$.

Thanks to the previous we can finally estimate the term on the right hand side of (3.17) as follows:

$$
\int_{\Omega} f_{n} u_{n}^{1-\gamma} \leq C^{1-\gamma}\|f\|_{L^{m}(\Omega)}\left(\int_{\Omega} \frac{1}{\delta^{(\gamma-1) m^{\prime}}}\right)^{\frac{1}{m^{\prime}}},
$$

which is finite since $\gamma<2-\frac{1}{m}$. This allows to have an estimate on $u_{n}$ in $W_{0}^{1, p}(\Omega)$ which is independent on $n$. Hence one can reason as in Step 2 of Theorem 3.2 in order to deduce the existence of a weak solution.

Finally we prove Theorem 3.5 .

Proof of Theorem 3.5 We choose $u_{n}$ itself as a test function in the weak formulation of (3.5) and applying the Hölder inequality and the Poincaré inequality, we get

$$
\begin{aligned}
\int_{\Omega}\left|\nabla u_{n}\right|^{p} & \stackrel{(32)}{\leq} \underline{C} \int_{\Omega} f u_{n}^{1-\gamma}+\bar{C}\|g\|_{L^{\infty}(\Omega)} \int_{\Omega} u_{n}^{p} \\
& \leq \underline{C}\|f\|_{L}\left(\frac{p^{*}}{1-\gamma}\right)_{(\Omega)}^{\prime}\left\|u_{n}\right\|_{L^{p^{*}(\Omega)}}^{1-\gamma}+\bar{C}\|g\|_{L^{\infty}(\Omega)} C_{p}^{p} \int_{\Omega}\left|\nabla u_{n}\right|^{p},
\end{aligned}
$$

which, recalling $1-\bar{C}\|g\|_{L^{\infty}(\Omega)} C_{p}^{p}>0$, implies that

$$
\int_{\Omega}\left|\nabla u_{n}\right|^{p} \leq \frac{\underline{C}}{1-\bar{C}|| g \|_{L^{\infty}(\Omega)} C_{p}^{p}}\|f\|_{L}\left(\frac{p^{*}}{1-\gamma}\right)_{(\Omega)}^{\prime}\left\|u_{n}\right\|_{L^{p^{*}(\Omega)}}^{1-\gamma} .
$$

Applying the Sobolev embedding Theorem in the right hand side of (3.18), we have

$$
\left\|u_{n}\right\|_{W_{0}^{1, p}(\Omega)}^{p} \leq \frac{\underline{C} \mathcal{S}^{1-\gamma}}{1-\bar{C}\|g\|_{L^{\infty}(\Omega)} C_{p}^{p}}\|f\|_{L}\left(\frac{p^{*}}{1-\gamma}\right)_{(\Omega)}^{\prime}\left\|u_{n}\right\|_{W_{0}^{1, p}(\Omega)}^{1-\gamma},
$$

where $\mathcal{S}$ is the constant of the embedding. Since $p>1-\gamma$ it follows that $\left\{u_{n}\right\}$ is bounded in $W_{0}^{1, p}(\Omega)$. So, up to subsequence, we have $u_{n} \rightarrow u$ weakly in $W_{0}^{1, p}(\Omega)$ and almost 
everywhere in $\Omega$. Finally we can repeat the argument of Step 2 of Theorem 3.4 in order to conclude that $u$ is a solution to (3.4).

\subsection{A Concluding Remark}

Here we underline that the result in Theorem 3.4 is not sharp, at least in the model case. Let $\Omega \subset \mathbb{R}^{N}$ be open and bounded with smooth boundary and let us consider the following problem

$$
\begin{cases}-\Delta u=\frac{f}{u^{\gamma}}+g u^{q} & \text { in } \Omega, \\ u=0 & \text { on } \partial \Omega,\end{cases}
$$

where $\gamma>1, q<1,0<f \in L^{1}(\Omega)$ and $g \in L^{\infty}(\Omega)$ nonnegative. We recall the following result proven in [39].

Theorem 3.8 Let $\gamma>1, q<1$ and let us suppose that there exists a function $u_{0} \in H_{0}^{1}(\Omega)$ such that

$$
\int_{\Omega} f u_{0}^{1-\gamma}<\infty
$$

Then there exists a solution $u \in H_{0}^{1}(\Omega)$ to (3.19).

Using the previous result we have the following existence theorem:

Theorem 3.9 Let $f \in L^{m}(\Omega)$ with $m>1$ be a nonnegative function and let $g \in L^{\infty}(\Omega)$ be a nonnegative function. Let $1<\gamma<3-\frac{2}{m}$ and $q<1$ then there exists a solution to problem (3.19).

Proof In order to show the existence of a solution we employ (3.20) with $u_{0}=\delta(x)^{t}$ for some $t>\frac{1}{2}$ and where $\delta(x)$ is the distance function from the boundary $\partial \Omega$. Indeed, one can show that an application of the Hölder inequality

$$
\int_{\Omega} f u_{0}^{1-\gamma} \leq C \int_{\Omega} \delta^{t(1-\gamma) m^{\prime}}
$$

and the last integral is finite thanks on the assumption $\gamma<3-\frac{2}{m}$.

We also remark that, in [16], Theorem 3.8 is extended for the case of the $p$-Laplacian operator with $p>2$. In this case one can show that a similar result to Theorem 3.9 with $1<\gamma<1+\frac{p(m-1)}{(p-1) m}$.

Funding Open Access funding provided by Università degli Studi di Napoli Federico II.

Open Access This article is licensed under a Creative Commons Attribution 4.0 International License, which permits use, sharing, adaptation, distribution and reproduction in any medium or format, as long as you give appropriate credit to the original author(s) and the source, provide a link to the Creative Commons licence, and indicate if changes were made. The images or other third party material in this article are included in the article's Creative Commons licence, unless indicated otherwise in a credit line to the material. If material is not included in the article's Creative Commons licence and your intended use is not permitted by statutory regulation or exceeds the permitted use, you will need to obtain permission directly from the copyright holder. To view a copy of this licence, visit http://creativecommonshorg/licenses/by/4.0/. 


\section{References}

1. Arcoya, D., Carmona, J., Leonori, T., Martínez-Aparicio, P.J., Orsina, L., Petitta, F.: Existence and nonexistence of solutions for singular quadratic quasilinear equations. J. Differ. Equ. 246, 4006-4042 (2009)

2. Arcoya, D., Martínez-Aparicio, P.J.: Quasilinear equations with natural growth. Rev. Mat. Iberoam. 24, 597-616 (2008)

3. Arcoya, D., Segura de León, S.: Uniqueness of solutions for some elliptic equations with a quadratic gradient term. ESAIM Control Optim. Calc. Var. 16, 327-336 (2010)

4. Boccardo, L., Murat, F.: Almost everywhere convergence of the gradients of solutions to elliptic and parabolic equations. Nonlinear Anal. 19, 581-597 (1992)

5. Boccardo, L., Casado-Diaz, J.: Some properties of solutions of some semilinear elliptic singular problems and applications to the $G$-convergence. Asymptot. Anal. 86, 1-15 (2014)

6. Boccardo, L., Orsina, L.: Sublinear elliptic equations in $l^{s}$. Houston Math. J 20, 99-114 (1994)

7. Boccardo, L., Orsina, L.: Semilinear elliptic equations with singular nonlinearities. Calc. Var. PDEs 37(3-4), 363-380 (2010)

8. Brandolini, B., Chiacchio, F., Trombetti, C.: Symmetrization for singular semilinear elliptic equations. Ann. Mat. Pura Appl. (4) 193(2), 389-404 (2014)

9. Brezis, H., Oswald, L.: Remarks on sublinear elliptic equations. Nonlinear Anal. 10, 55-64 (1986)

10. Canino, A., Esposito, F., Sciunzi, B.: On the höpf boundary lemma for singular semilinear elliptic equations. J. Diff. Equ. 266(9), 5488-5499 (2019)

11. Canino, A., Sciunzi, B., Trombetta, A.: Existence and uniqueness for $p$-Laplace equations involving singular nonlinearities. NoDEA Nonlinear Differ. Equ. Appl. 23, 8 (2016)

12. Carmona, J., Martínez-Aparicio, P.J.: A singular semilinear elliptic equation with a variable exponent. Advanced Nonlinear Studies 16, 491-498 (2016)

13. Cîrstea, F., Ghergu, M., Rădulescu, V.: Combined effects of asymptotically linear and singular nonlinearities in bifurcation problems of Lane-Emden-Fowler type. J. Math Pures Appl. (9) 84(4), 493-508 (2005)

14. Coclite, G.M., Coclite, M.M.: On the summability of weak solutions for a singular Dirichlet problem in bounded domains. Adv. Differ. Equ. 19(5-6), 585-612 (2014)

15. Coclite, M.M., Palmieri, G.: On a singular nonlinear Dirichlet problem. Comm. Partial Differ. Equ. 14(10), 1315-1327 (1989)

16. Cong, S., Han, Y.: Compatibility conditions for the existence of weak solutions to a singular elliptic equation. Bound. Value Probl. 2015 27, 11 (2015)

17. Crandall, M.G., Rabinowitz, P.H., Tartar, L.: On a dirichlet problem with a singular nonlinearity. Comm. Part. Diff. Eq. 2(2), 193-222 (1977)

18. De Cave, L.M., Oliva, F.: Elliptic equations with general singular lower order term and measure data. Nonlinear Anal. 128, 391-411 (2016)

19. De Cave, L.M., Durastanti, R., Oliva, F.: Existence and uniqueness results for possibly singular nonlinear elliptic equations with measure data. Nonlinear Differ. Equ. Appl. 25, 18 (2018)

20. Durastanti, R.: Asymptotic behavior and existence of solutions for singular elliptic equations. Annali di Matematica 199, 925-954 (2020)

21. Diaz, J.I., Rakotoson, J.M.: On the differentiability of very weak solutions with right-hand side data integrable with respect to the distance to the boundary. J. Funct. Anal. 257, 807-831 (2009)

22. Faraci, F., Smyrlis, G.: Three solutions for a singular quasilinear elliptic problem. Proc. Edinb. Math. Soc. (2) 62(1), 179-196 (2019)

23. Giachetti, D., Martínez-Aparicio, P.J., Murat, F.: A semilinear elliptic equation with a mild singularity at $\mathrm{u}=0$ : Existence and homogenization. J. Math. Pures Appl. 107, 41-77 (2017)

24. Giachetti, D., Martínez-Aparicio, P.J., Murat, F.: Definition, existence, stability and uniqueness of the solution to a semilinear elliptic problem with a strong singularity at $u=0$. Ann. Scuola Normale Pisa (5) 18(4), 1395-1442 (2018)

25. Giachetti, D., Petitta, F., Segura de León, S.: A priori estimates for elliptic problems with a strongly singular gradient term and a general datum. Differ. Integral Equ. 26(9/10), 913-948 (2013)

26. Godoy, T., Guerin, A.: Existence of nonnegative solutions to singular elliptic problems, a variational approach. Discrete Contin. Dyn. Syst. 38(3), 1505-1525 (2018)

27. Gui, C., Lin, F.: Regularity of an elliptic problem with a singular nonlinearity. Proc. Roy. Soc. Edinburgh Sect. A 123(6), 1021-1029 (1993)

28. Leray, J., Lions, J.L.: Quelques résulatats de višik sur les problémes elliptiques nonlinéaires par les méthodes de Minty-Browder. Bull. Soc. Math. France 93, 97-107 (1965) 
29. Lazer, A.C., McKenna, P.J.: On a singular nonlinear elliptic boundary-value problem. Proc. Amer. Math. Soc. 111(3), 721-730 (1991)

30. Loc, N.H., Schmitt, K.: Boundary value problems for singular elliptic equations, Rocky Mountain J. Math 41 (2), 555-572 (2011)

31. Oliva, F.: Regularizing effect of absorption terms in singular problems. J. Math. Anal. Appl. 472(1), 1136-1166 (2019)

32. Oliva, F., Petitta, F.: On singular elliptic equations with measure sources. ESAIM Control Optim Calc Var 22, 289-308 (2016)

33. Oliva, F., Petitta, F.: Finite and infinite energy solutions of singular elliptic problems: existence and uniqueness. J. Differ. Equ. 264(1), 311-340 (2018)

34. Sakaguchi, S.: Concavity properties of solutions to some degenerate quasilinear elliptic Dirichlet problems. Ann. Scuola Norm. Sup. Pisa Cl. Sci. 14, 403-421 (1987)

35. Santos, C.A., Santos, L.: How to break the uniqueness of $w_{\mathrm{loc}}^{1, p}(\Omega)$-solutions for very singular elliptic problems by non-local terms. Z. Angew. Math. Phys. 69(6), Art. 14522 (2018)

36. Shi, J., Yao, M.: On a singular nonlinear semilinear elliptic problem. Proc. Roy. Soc. Edinburgh Sect. A 128(6), 1389-1401 (1998)

37. Stampacchia, G.: Le problème de Dirichlet pour les équations elliptiques du seconde ordre à coefficients discontinus. Ann. Inst. Fourier (Grenoble) 15, 189-258 (1965)

38. Stuart, C.A.: Existence and approximation of solutions of nonlinear elliptic problems, Mathematics Report, Battelle Advanced Studies Center, Geneva, Switzerland (1976)

39. Sun, Y.: Compatibility phenomena in singular problems. Proc. Roy. Soc. Edinburgh Sect. A 143(6), 1321-1330 (2013)

Publisher's Note Springer Nature remains neutral with regard to jurisdictional claims in published maps and institutional affiliations. 